\title{
Entropy of a Free Quantum Particle
}

\author{
Jian-Ping Peng \\ Department of Physics, Shanghai Jiao Tong University, Shanghai, China \\ Email: jppeng@sjtu.edu.cn
}

Received September 30, 2012; revised November 2, 2012; accepted November 10, 2012

\begin{abstract}
The time-dependent entropy of a single free quantum particle in the non-relativistic regime is studied in detail for the process started from a fully coherent quantum state to thermodynamic equilibrium with its surroundings at a finite temperature. It is shown that the entropy at the end of the process converges to a universal constant, as a result of thermal interaction.
\end{abstract}

Keywords: Entropy Generation; Quantum Thermodynamic Systems

\section{Introduction}

It is well-known that entropy, as the measure of "the amount of uncertainty", can not decrease in any spontaneous process according to the second law of thermodynamics [1]. In a recent work [2], we studied the thermodynamics of a single quasi-free massive quantum particle, by performing statistics directly on the matter wave of the particle. Taking into account the detailed configuration of diffraction in real space and thermal interaction with the surround space at a finite temperature, the complicated behavior of the time-dependent internal energy is studied for the whole process started from a fully coherent quantum state to thermodynamic equilibrium with the surrounding space. An expression for the entropy of the particle is also shown in [2]. The purpose of the present article is to present the detailed derivation of the expression of the time-dependent entropy for the particle and study in more detail the physics in the irreversible process. Numerical calculations confirm that the entropy increases monotonically with time and the entropy generated in the whole process converges to a universal constant. Although the system studied here is the simplest quantum system at a finite temperature, it already shows how a single quantum particle feels the temperature of its surrounding space. In conventional quantum mechanics, entropylike concepts are defined only for statistical description of ensembles of identical quantum systems. Our results here confirm the conclusion that entropy is a physical observable that can be well-defined for each individual quantum system at finite temperatures [3].

\section{Model Calculations}

The system considered is a structureless quantum particle of mass $m$ and kinetic energy $E_{0}$ initially at the origin, moving along the $x$-axis in a space at a nonzero temperature $T$. The space here may be filled with electromagnetic radiation just as the cosmic background in the universe. In quantum mechanics, the particle is described by a wave-packet sharply peaked at the de Broglie wavelength $\lambda=h /\left(2 m E_{0}\right)^{1 / 2}$ and the wave-packet propagates at group velocity $V_{g}=h /(m \lambda)$, with $h$ being the Planck's constant. The matter wave front is assumed to be circular with finite radius $a_{0}$ which is large compared with the wavelength, so that the shape and linear dimension of the forward-going wave-front remains unchanged. Strictly speaking, the particle is quasi-free in the model calculation, even though there is no interaction with other particles. If the radius $a_{0}$ tends to be infinitely large, the results reduce to that of a free quantum particle. A point in the central part of the wave-front generates forward-going semi-spherical waves, according to the Huygens principle. A point at the edge of the wave-front is assumed to generate out-going fully spherical waves and thus the particle undergoes a kind of reflection. The kinetic energy associated with the forward-going wave-packet follows the form [2]

$$
E_{k}(x)=E_{0} \exp \left(-2 \lambda x / a_{0} L\right),
$$

where $x=V_{g} t$ representing its central position and $L$ is a temperature dependent parameter of dimension length and is expected to be infinitely large as the temperature tends to zero. This is just the energy for the source to generate out-going fully spherical waves. In general, all energy states are not equally likely. In principle, the particle may be in any of these diffracted states besides the forward-going plane-wave state, i.e., the particle itself constitutes automatically a thermodynamic system as a 
result of diffraction at the edge of its matter-wave front. Thermal interaction between the particle's system and the surrounding space becomes possible and the space here acts as the heat reservoir at constant temperature. As time goes on, the probability decreases for the particle in the quantum state described by forward-going wavepacket. At the end of the process, the particle can only be in a series of states diffracted at the edge and moves equally to all directions. The partition function at a given time is written in the form $Z(t)=Z_{f}(t)+Z_{d}(t)$, with

$$
Z_{f}(t)=\exp \left(-\frac{2 \lambda V_{g} t}{a_{0} L}\right) \exp \left[-\beta E_{0} \exp \left(-\frac{2 \lambda V_{g} t}{a_{0} L}\right)\right]=\exp \left(-\beta E_{0} \mathrm{e}^{-t_{r}}-t_{r}\right)
$$

representing contribution from the forward-going wave-front, and

$$
Z_{d}(t)=\int_{0}^{V_{g} t} P_{E}(x) \exp \left[-\beta E_{0} d_{0} P_{E}(x)\right] \mathrm{d} x=\left[\mathrm{e}^{-Z_{0} \exp \left(-t_{r}\right)}-\mathrm{e}^{-Z_{0}}\right] / Z_{0}
$$

from all spherical waves diffracted at the edge, respectively. Here the notation $\beta=1 / k_{B} T$ is used as usual with $k_{B}$ being the Boltzmann constant. The constant $Z_{0}$ is defined as the non-zero real solution of the transcend equation $\exp \left(Z_{0}\right)-2 Z_{0}-1=0$, and an approximate value $Z_{0}=1.25643$ is used in our numerical calculations. The probability density function is defined as

$$
P_{E}(x)=\left(2 \lambda / a_{0} L\right) \exp \left(-2 \lambda x / a_{0} L\right)
$$

and the step length is chosen to be $d_{0}=Z_{0} a_{0} L /\left(2 \lambda \beta E_{0}\right)$. The time is scaled as $t_{r}=t / t_{c}$, where $t_{c}=m a_{0} L /(2 h)$ is the temperature dependent characteristic time.

The expectation value of energy of the particle or its internal energy $U_{x}(t)$ for the coordinate is

$$
\begin{aligned}
U_{x}(t) & =\frac{1}{Z(t)} \int_{0}^{V_{g} t} E_{0} d_{0} P_{E}^{2}(x) \exp \left[-\beta E_{0} d_{0} P_{E}(x)\right] \mathrm{d} x+\frac{E_{0}}{Z(t)} \exp \left(-4 \lambda V_{g} t / a_{0} L\right) \exp \left[-\beta E_{0} \exp \left(-2 \lambda V_{g} t / a_{0} L\right)\right] \\
& =\frac{\exp \left(-t_{r}-Z_{0} \mathrm{e}^{-t_{r}}\right)-\exp \left(-Z_{0}\right)+\beta E_{0} \exp \left(-\beta E_{0} \mathrm{e}^{-t_{r}}-2 t_{r}\right)}{\beta Z(t)}+\frac{\exp \left(-Z_{0} \mathrm{e}^{-t_{r}}\right)-\exp \left(-Z_{0}\right)}{\beta Z_{0} Z(t)},
\end{aligned}
$$

which evolves depending on the temperature and the particle's initial energy in a complicated form. In general, a quantum particle absorbs or gives out heat continuously when its initial energy $E_{0}$ is less or more than $k_{B} T / 2$, indicating exchange of energy between the particle and its surrounding space in the whole process. The limiting value of the internal energy for the freedom in the $x$-direction is $k_{B} T / 2$, regardless of its initial energy [2]. The limit is reached within several $t_{c}$ and the overall decay or increase in internal energy does not follow the simple exponential form.

We use the well-known definition of entropy of a discrete system

$$
S=-k_{B} \sum_{i} p_{i} \ln p_{i}
$$

where $p_{i}$ is the probability in the $i^{\text {th }}$ state and the sum goes over all states accessible to the system. The definition is perfectly unambiguous for systems of any size and there is no restriction to equilibrium situations: the probability will be time-dependent if the system evolves dynamically. The entropy of the particle is thus a function of the probability distribution and is not fluctuating since it has nothing to do with the state in which it happens to be. We first assume that the forward-going wave-front representing a single quantum state makes little contribution to the entropy. The entropy is then determined by all those states diffracted at the edge. In quantum mechanics, the spreads in energy and time are related by the uncertainty relation $\Delta E \cdot \Delta t \geq h / 2 \pi$. To prepare the original state with energy $E_{0}$, the uncertainty in time is estimated to be $\Delta t \approx h / 2 \pi E_{0}$, corresponding to an uncertainty in position of the particle $V_{g} \Delta t \approx \lambda$. Therefore, waves diffracted at the edge should be indistinguishable when the wave-front moves forward within about one wavelength. Please note that the analysis here does not mean the entropy is physically related to the principle of uncertainty in quantum mechanics. In fact, as shown below, a different choice of the indistinguishable length leads to an unimportant additive constant in entropy. We choose $\lambda$ as the shortest step and rewrite the partition function due to diffraction at the edge of the wave-front in discrete form

$$
Z_{d}(t)=\sum_{l=0}^{V_{g} t / \lambda} A \lambda f_{l} \mathrm{e}^{-Z_{0} f_{l}}
$$

where $f_{l}=\exp (-A \lambda l)$ and $A=\left(2 \lambda / a_{0} L\right)$. According to Equation (5), the entropy is then 


$$
S_{x d}(t)=-\frac{k_{B}}{Z_{d}(t)} \sum_{l=0}^{V_{g} t / \lambda} A \lambda f_{l} \mathrm{e}^{-Z_{0} f_{l}} \ln \frac{A \lambda f_{l} \mathrm{e}^{-Z_{0} f_{l}}}{Z_{d}(t)},
$$

where the subscript $x$ represents the coordinate and $d$ diffracted states at the edge. This expression is exact and must be used within the time $t \leq \lambda / V_{g}$. At a later time $t \gg \lambda / V_{g}$, by changing summation into integration and with the help of the exponential integral function [4], we obtain

$$
\begin{aligned}
\frac{S_{x d}(t)}{k_{B}}= & 1+\ln Z_{d}(t)+\frac{\exp \left(-t_{r}-Z_{0} \mathrm{e}^{-t_{r}}\right)-\exp \left(-Z_{0}\right)}{Z_{d}(t)} \\
& +\frac{t_{r} \exp \left(-Z_{0} \mathrm{e}^{-t_{r}}\right)+E i\left(-Z_{0} \mathrm{e}^{-t_{r}}\right)-E i\left(-Z_{0}\right)}{Z_{0} Z_{d}(t)} .
\end{aligned}
$$

Note that the constant term $\ln (A \lambda)$ is eliminated because it is an additive constant depending on how to define a distinguishable state and in thermodynamics we are interested only in the entropy generation during the process of evolution. Moreover, the factor $A \lambda$ in the discrete form of the partition function Equation (6) corresponds to a common weight factor for all states in the problem and has no physical consequence in calculating the average value of a quantity except the entropy.

Similar discussion applies also to the case that the contribution from the forward-going wave-front is included. The entropy of the particle is expressed as

$$
\begin{aligned}
S_{x}(t)= & -\frac{k_{B}}{Z(t)} \sum_{l=0}^{V_{g} t / \lambda} A \lambda f_{l} \mathrm{e}^{-Z_{0} f_{l}} \ln \frac{A \lambda f_{l} \mathrm{e}^{-Z_{0} f_{l}}}{Z(t)} \\
& -k_{B} \frac{Z_{f}(t)}{Z(t)} \ln \frac{Z_{f}(t)}{Z(t)},
\end{aligned}
$$

which ensures that the entropy starts from zero and remains positive later. At a time $t \gg \lambda / V_{g}$, by eliminating the $\ln (A \lambda)$ term once again and changing the summation into integration, the time-dependent entropy of the particle for the $x$-coordinate can be obtained

$$
\begin{aligned}
& \frac{S_{x}(t)}{k_{B}}=\beta U_{x}(t)+\ln Z(t)+\frac{1}{Z(t)} \\
& \times\left\{\int_{1}^{\exp \left(-t_{r}\right)} \mathrm{e}^{-Z_{0} l} \ln l \mathrm{~d} l+t_{r} \exp \left[-t_{r}-\beta E_{0} \exp \left(-t_{r}\right)\right]\right\} .
\end{aligned}
$$

In fact, time-dependent entropy of a quantum system has been studied by Gheorghiu-Svirschevski using an extended Liouville-von Neumann equation [5]. Unfortunately, we failed to obtain an expression for the entropy using this theory to compare with Equation (10). The reason is that the system here is not described by standard plane-wave with infinite spacial extension. The present work starts from a circular matter wave pulse with finite radius $a_{0}$, its time evolving is governed by the Huy-
gens-Fresnel principle. Furthermore, every point at the edge of a wave-front is assumed to generate continuously out-going spherical secondary wavelets. In principle, such a system can be studied with the path integral formulation of quantum theory, but not the Schroedinger formulation.

In Figure 1 we plot the numerical results for the entropy of the particle determined by Equation (10) as a function of the scaled time for different values of $\beta E_{0}$. For $\beta E_{0} \leq 2$, the entropy starts from zero and increases with increasing $\left(t / t_{c}\right)$ and shows little dependence on the exact value of $\beta E_{0}$. For $2<\beta E_{0} \leq 50$, Equation (10) may become negative numerically, indicating that it is inexact in the initial stage. Fortunately, it starts to be positive at a latter time $\left(t / t_{c}\right)<1$. For $\beta E_{0} \geq 50$, the entropy starts to be non-negative at $\left(t / t_{c}\right)=1$ and then increases monotonically with time in accordance with the second law of thermo-dynamics. Mathematically, Equation (10) reduces to Equation (8) in the limit of large $\beta E_{0}$ and thus the entropy as a function of the scaled time $\left(t / t_{c}\right)$ shows no direct dependence on $\beta E_{0}$.

Although the entropy evolves in a complicated from, it tends to reach its limit within a time about $\left(t / t_{c}\right) \approx 5$. The limit can be calculated exactly from Equation (10) and is of the form

$$
\begin{aligned}
S_{x}(\infty) & =k_{B}\left[\frac{1}{2}+\ln \frac{1-\mathrm{e}^{-Z_{0}}}{Z_{0}}+\frac{C+\ln Z_{0}-E i\left(-Z_{0}\right)}{1-\mathrm{e}^{-Z_{0}}}\right] \\
& \approx 1.27 k_{B}
\end{aligned}
$$

where $E i$ the exponential integral function [4]. Note that the limit is universal with no regards to the unknown

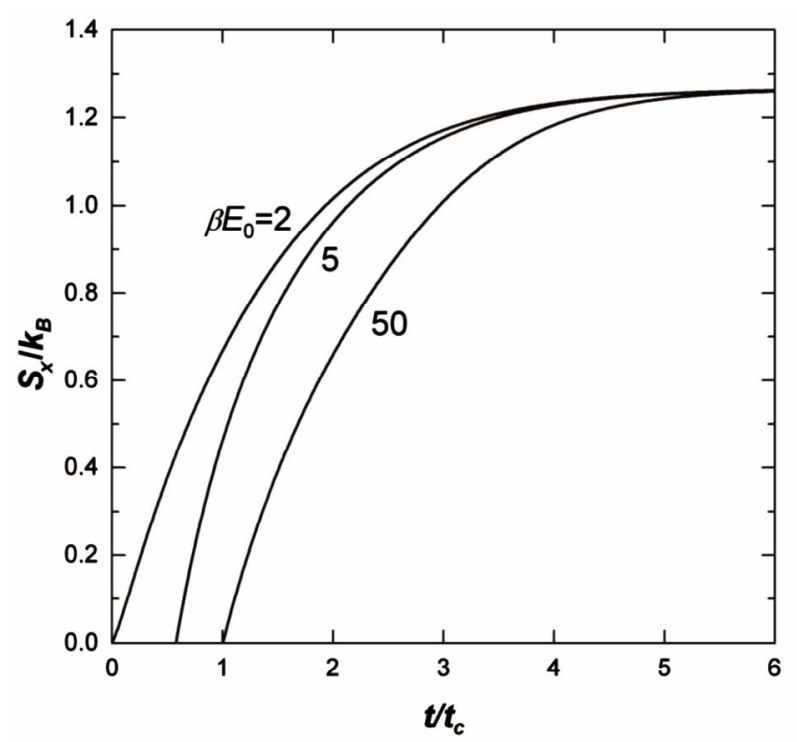

Figure 1. The entropy of a single quantum particle for the $x$-coordinate as a function of the scaled time for different values of $\beta E_{0}$. The curves may become inexact for $\left(t t_{c}\right)<1$, as described in the text. 
parameter $L$, the temperature of the surrounding space and the initial state of the particle. In a textbook of statistical physics, it is well-known that the entropy per particle in an ideal gas of $N$ particles in a volume $V$ and at constant temperature $T$ is expressed as the Sackur-Tetrode formula [6]

$$
\frac{S}{N}=k_{B} \ln \frac{N}{V}+\frac{5}{2} k_{B}+\frac{3}{2} k_{B} \ln \frac{2 \pi m k_{B} T}{h^{2}},
$$

showing dependences on the temperature and the mass of the particle. It should be pointed that Equation (11) is the entropy per freedom generated in the process started from an initial coherent quantum state to final thermodynamic equilibrium with its surroundings, before we call it a particle in conventional statistical physics, i.e., it moves equally to all directions. For a real free particle $\left(a_{0} \gg \lambda\right)$, independent motion is allowed in the perpendicular direction. Therefore, the total entropy per particle generated in the whole process of decoherence should be $3 S_{X}(\infty) \approx 3.81 k_{B}$.

\section{Conclusion}

In conclusion, we have derived an expression for the time-dependent entropy of a single non-relativistic quantum particle freely moving in a space at constant nonzero temperatures. The entropy increases monotonically with time in accordance with the second law of thermodynamics. Although the initial state of the particle and the temperature of the surrounding space play important roles in the process, the total entropy generated tends to be a universal constant.

\section{REFERENCES}

[1] L. D. Landau and E. M. Lifshitz, "Statistical Physics," 3rd Edition, Pergamon Press Ltd., Oxford, 1980.

[2] J. P. Peng, "Temperature Dependent Motion of a Massive Quantum Particle," Journal of Modern Physics, Vol. 3, 2012, pp. 610-614. doi:10.4236/jmp.2012.37083

[3] G. P. Beretta, "Entropy and Irreversibility for a Single Isolated Two Level System: New Individual Quantum States and New Nonlinear Equation of Motion," International Journal of Theoretical Physics, Vol. 24, No. 2, 1985, pp. 119-134. doi:10.1007/BF00672647

[4] I. S. Gradshteyn and I. M. Rizhik, "Tables of Integrals, Series, and Products," 7th Edition, Elservier Inc., London, 2007.

[5] S. Gheorghiu-Svirschevski, "Nonlinear Quantum Evolution with Maximal Entropy Production," Physical Review A, Vol. 63, 2001, Article ID: 022105.

[6] M. Plischke and B. Bergersen, "Equilibrium Statistical Physics," 2nd Edition, World Scientific Publishing Co. Pte. Ltd., 2003. 